\title{
Parameter Derivation of Type-2 Discrete-Time Phase-Locked Loops Containing Feedback Delays
}

\author{
Joey Wilson, Andrew Nelson, and Behrouz Farhang-Boroujeny \\ joey.wilson@utah.edu,nelson@math.utah.edu, farhang@ece.utah.edu \\ University of Utah \& L-3 Communications
}

\begin{abstract}
Modern implementations of discrete-time phaselocked loops (DT-PLLs) often contain delayed feedback. The delays are usually a side effect to pipelining, filtering, or other inner-loop mechanisms. Each delay increases the order of the system by introducing an additional pole to the closed-loop transfer function, and in many cases, makes the traditional type2 loop equations obsolete. This paper describes how the secondorder notions of damping and natural frequency can be applied to type-2 DT-PLLs in the presence of any number of delays. It provides equations for loop parameters that will provide a desired transient behavior based on damping and natural frequency, along with a test to ensure the accuracy of the results. The novelty of this work is that loop parameters can be found in closedform and ensured to be accurate, without the need for human interaction, simulations, or numerical root-finding algorithms.
\end{abstract}

Index Terms-Phase locked loops, Delay effects, Reduced order systems, Dominant Poles

\section{INTRODUCTION}

With the advent of the software radio and other modern digital devices, discrete-time phase-locked loops (DT-PLLs) are being implemented more than ever before. These modern implementations often contain delayed feedback, which is usually a side effect to pipelining, filtering, or other innerloop mechanisms. Each sample delay introduces a pole into the closed-loop transfer function, resulting in a high-order system. The delays limit the loop's stability regions, change its transient behavior, and in many cases render the traditional second-order analysis obsolete. It is not uncommon today to encounter loops with delays on the order of tens to hundreds.

The effects of such delays on the stability and phase margin for DT-PLLs with delays have been previously investigated [1], [2], and [3]. The effect of delays on stability, root-locus, and frequency-response, and investigation of some specific scenarios with a low number of delays is covered in [4].

Other works have used optimization approaches to address the presence of delays. A Weiner approach to optimizing the loop filter in steady-state with the presence of delays is found in [5]. Previous work also showed that the type-II discretetime PLL has the same structure as a Kalman filter, and a modified version has been proposed to compensate for loop delay [6].

A PLL designer is often concerned with finding loop parameters $K_{p}$ and $K_{i}$ (as shown in Fig. 1) to achieve a particular transient behavior. Transient behavior is often described by the second-order notions of damping factor $\zeta$ and natural frequency $\omega_{n}$. [1], [2], and other references on DT-PLLs

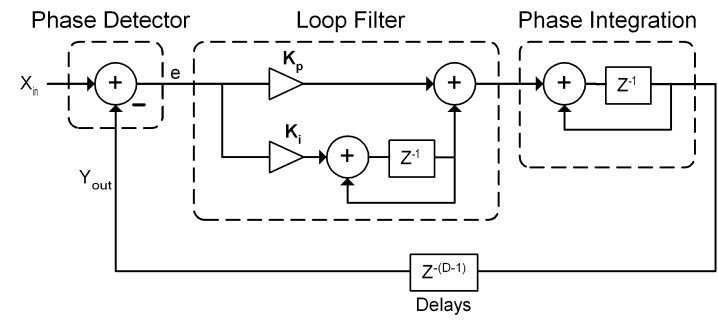

Fig. 1. Phase domain model of type-2 DT-PLL with PPI filter

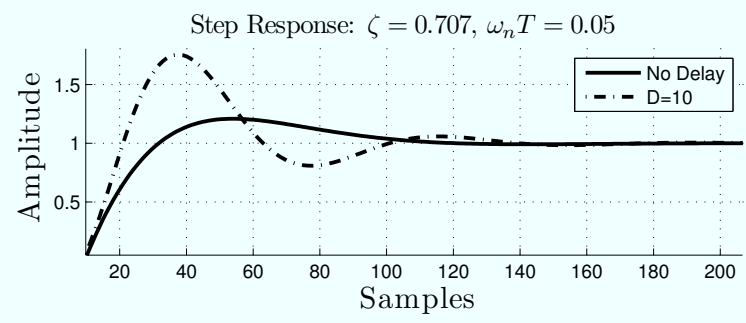

Fig. 2. An example illustrating the inaccuracy of using traditional loop parameter equations in the presence of delays. Here, loop parameter values for $K_{p}$ and $K_{i}$ are calculated from (2), regardless of the number of delays.

use the following second-order definitions, regardless of the number of delays.

$$
\omega_{n} T=\sqrt{K_{i}} \quad \text { and } \quad \zeta=\frac{K_{p}}{2 \sqrt{K_{i}}}
$$

where $T$ is the sample time interval. Equivalently,

$$
K_{p}=2 \omega_{n} T \zeta \quad \text { and } \quad K_{i}=\left(\omega_{n} T\right)^{2} .
$$

The derivation of (1) can be found in [4].

Ignoring the delays often results in inaccurate prediction of transient behavior, especially at high $\omega_{n} T$ values [7]. For example, using (2) to achieve a damping factor $\zeta=.707$ and $\omega_{n} T=.05$ in the presence of 10 sample delays will yield loop parameters $K_{p}$ and $K_{i}$ that do not provide a response with the expected characteristics (See Fig. 2).

Modern communication systems are designed to adapt their modulation, data rates, and other configurations under various conditions. With this flexibility comes the challenge of ensuring that loop parameters are accurate for proper tracking and signal acquisition. This is complicated further when delayed feedback is introduced, since stability regions are severely reduced. Even slight changes in loop parameters may become problematic in high-delay cases. 
While it is true that numerical software can easily find the roots of high-order systems and plot transient responses for DT-PLLs with delays, this approach is limited for the following reasons.

- In most cases, plotting and interpreting transient responses for a given set of loop parameters requires a human's judgment. This prevents automation, since the parameters are calculated by a human on a device other than the system of interest.

- By taking an empirical approach to choosing loop gains, designers must guess-and-check parameters, iterating until satisfactory values are found. This can require a significant amount of time, especially when the stability regions of a loop are very small. Since it is common for communication systems to have many sets of parameters, the problem of time consumption is further amplified.

It is therefore desirable to form analytic solutions relating the desired loop characteristics to loop parameters, without the need for human interaction. This paper derives equations for accurately determining loop parameters $K_{p}$ and $K_{i}$ in the presence of delays, based on a desired damping factor $\zeta$, and natural frequency $\omega_{n}$. A numerical test is provided in Section V that can be used to ensure that the results are accurate, without the need for human interaction, simulations, or numerical root-finding algorithms.

The reduction of a DT-PLL with delays to a second-order system is possible through the use of dominant poles, a commonly tool used to reduce higher-order systems [8], [9], [10]. Past literature on this subject is mostly concerned with the selection of dominant poles in a higher-order system. This paper, however, assumes that the dominant poles are known, and the system is derived around this assumption. Using tools from complex analysis, as shown in Section V, the accuracy of the dominant pole assumption is tested and verified in the z-domain.

\section{Phase Domain Model}

Fig. 1 is the phase domain model of a type-2 DT-PLL with a total of $D$ delays [4]. This particular model includes the most common loop filter architecture known as the proportionalplus-integral (PPI) filter. The phase domain model is not used to implement a PLL, but is derived as a linear model used for analysis. The delays have been grouped into one $z^{-(D-1)}$ term at the end of the feedback path. One additional delay occurs in the phase integration element, resulting in a total of $D$ feedback delays. The closed-loop transfer function for the circuit shown in Fig. 1 is

$$
\frac{Y_{\text {out }}}{X_{\text {in }}}=\frac{K_{p} z-K_{p}+K_{i}}{z^{D+1}-2 z^{D}+z^{D-1}+K_{p} z+K_{i}-K_{p}} .
$$

Since the first three terms of the denominator do not involve the terms $K_{p}$ and $K_{i}$, they are grouped into one function defined as

$$
C(z)=z^{D+1}-2 z^{D}+z^{D-1} .
$$

The stability and transient behavior of the loop is determined by the roots of the characteristic polynomial

$$
P(z)=C(z)+K_{p} z-K_{p}+K_{i},
$$

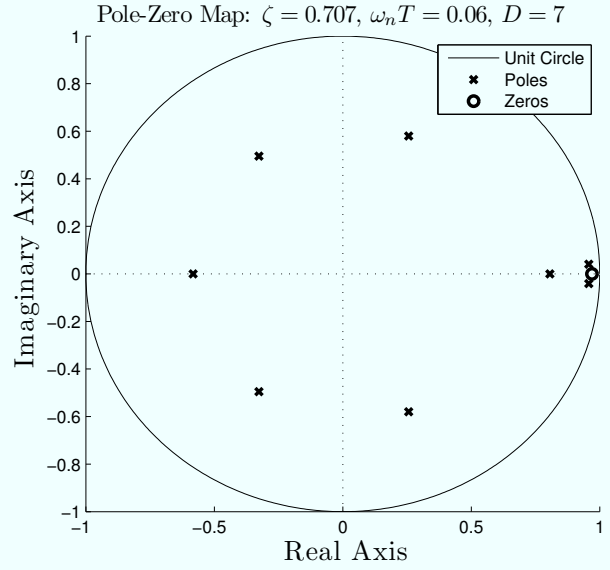

Fig. 3. A typical pole-zero plot for a DT-PLL with delays. Dominant poles can be seen near the right hand edge of the circle.

which is the denominator of the closed-loop transfer function (3). The order of the system is $D+1$, and when $D=1$, the system reduces to second-order. $D$ can never be less than one, as any realizable digital loop must contain at least one delay.

\section{Dominant Poles}

Dominant poles are commonly used to reduce a high-order system to that of a second-order approximation [8], [9], [10]. This is useful because damping ratio and natural frequency, which describe transient behavior, are defined as second-order characteristics. A common criteria for dominance in the sdomain is that any pole $A$ times farther from the $j \omega$-axis than the closest (dominant) poles can be ignored. The value of $A$ can be increased arbitrarily to make the approximation stronger, but good results were found in this study with $A=3$. This is comparable and in accordance with previous work on the study of PLL stability limits [7].

There is a one-to-one mapping of natural frequency and damping to the location of the poles of a continuous-time second-order system. This mapping is defined in control literature [4], [10] as

$$
H(s)=\frac{2 \zeta \omega_{n} s+\omega_{n}^{2}}{s^{2}+2 \zeta \omega_{n} s+\omega_{n}^{2}}
$$

where $\zeta$ is the damping factor and $\omega_{n}$ is the natural frequency.

Using the roots of the denominator and the transformation $z=e^{s T}$ yields the $\mathrm{z}$-domain pole locations

$$
z_{0,1}=e^{-\omega_{n} T\left(\zeta \pm \sqrt{\zeta^{2}-1}\right)} .
$$

In the z-domain, any pole with magnitude less than the magnitude of the dominant-poles raised to the $A^{\text {th }}$ power may be ignored. See Fig. 3 for an example.

With the correct choice of $K_{p}$ and $K_{i}$, it is possible to set two roots of the high-order characteristic polynomial (5) such that they match the second-order z-domain poles. This is a key concept in the dominant-pole approximation of a DTPLL with delays. The following section derives equations for proper selection of $K_{p}$ and $K_{i}$. 
IV. Determining Loop Parameters $K_{p}$ And $K_{i}$

In this section, it is assumed that the dominant pole criteria is satisfied and the loop is stable (the validity of this assumption will be addressed in Section V). Loop parameters $K_{p}$ and $K_{i}$ can be derived to ensure that 2 of the $D+1$ poles are placed in the correct locations for a desired dominant damping and dominant natural frequency. Three different derivations are used for the cases of an underdamped, critically damped, or overdamped system.

\section{A. Underdamped $(\zeta<1)$}

When a system is underdamped, two poles exist as complex conjugates. Following (7), they are defined as

$$
\begin{aligned}
z_{0,1} & =z_{r} \pm j z_{i}=r e^{ \pm j \theta} \\
R & =e^{-\omega_{n} T \zeta} \\
\theta & =\omega_{n} T \sqrt{1-\zeta^{2}} \\
z_{r} & =R \cos (\theta) \\
z_{i} & =R \sin (\theta) .
\end{aligned}
$$

Plugging $z_{0}$ into the characteristic polynomial (5) and equating to zero results in

$$
C\left(z_{0}\right)+K_{p} z_{0}-K_{p}+K_{i}=0 .
$$

Substituting $z_{0}=z_{r}+j z_{i}$ and setting the real and imaginary parts equal to zero results in the linear system:

$$
\left[\begin{array}{cc}
z_{r}-1 & 1 \\
z_{i} & 0
\end{array}\right]\left[\begin{array}{c}
K_{p} \\
K_{i}
\end{array}\right]=\left[\begin{array}{c}
-\Re\left[C\left(z_{0}\right)\right] \\
-\Im\left[C\left(z_{0}\right)\right]
\end{array}\right]
$$

where

$$
\begin{aligned}
\Re\left[C\left(z_{0}\right)\right]= & R^{D+1} \cos (\theta(D+1))-2 R^{D} \cos (\theta D) \\
& +R^{D-1} \cos (\theta(D-1)),
\end{aligned}
$$

and

$$
\begin{aligned}
\Im\left[C\left(z_{0}\right)\right]= & R^{D+1} \sin (\theta(D+1))-2 R^{D} \sin (\theta D) \\
& +R^{D-1} \sin (\theta(D-1)) .
\end{aligned}
$$

Since $z_{i}, z_{r}$, and $C\left(z_{0}\right)$ are known, the loop parameters are determined by

$$
\left[\begin{array}{c}
K_{p} \\
K_{i}
\end{array}\right]=\frac{1}{z_{i}}\left[\begin{array}{cc}
0 & 1 \\
z_{i} & 1-z_{r}
\end{array}\right]\left[\begin{array}{l}
-\Re\left[C\left(z_{0}\right)\right] \\
-\Im\left[C\left(z_{0}\right)\right]
\end{array}\right] .
$$

\section{B. Overdamped $(\zeta>1)$}

When a system is overdamped, two distinct poles exist on the real axis:

$$
z_{0,1}=e^{-\omega_{n} T\left[\zeta \pm \sqrt{\zeta^{2}-1}\right]} .
$$

Using these two values in the characteristic polynomial (5) and equating to zero, the following equation results.

$$
C\left(z_{i}\right)+K_{p} z_{i}-K_{p}+K_{i}=0
$$

for $i=0,1$ which can be expressed as

$$
\left[\begin{array}{cc}
z_{0}-1 & 1 \\
z_{1}-1 & 1
\end{array}\right]\left[\begin{array}{c}
K_{p} \\
K_{i}
\end{array}\right]=\left[\begin{array}{l}
-C\left(z_{0}\right) \\
-C\left(z_{1}\right)
\end{array}\right] .
$$

Solving for $K_{p}$ and $K_{i}$ yields

$$
\left[\begin{array}{c}
K_{p} \\
K_{i}
\end{array}\right]=\frac{1}{z_{0}-z_{1}}\left[\begin{array}{cc}
1 & -1 \\
1-z_{1} & z_{0}-1
\end{array}\right]\left[\begin{array}{l}
-C\left(z_{0}\right) \\
-C\left(z_{1}\right)
\end{array}\right]
$$

\section{Critically Damped $(\zeta=1)$}

When a system is critically damped, a pole of multiplicity two exists on the real axis:

$$
z_{0,1}=e^{-\omega_{n} T} .
$$

Both the characteristic polynomial (5) and its derivative at this value must be equal to zero. This leads to

$$
\begin{gathered}
C\left(z_{0}\right)+K_{p} z_{0}-K_{p}+K_{i}=0 \\
C^{\prime}\left(z_{0}\right)+K_{p}=0,
\end{gathered}
$$

where

$$
C^{\prime}(z)=\frac{d C}{d z}=(D+1) z^{D}-2 D z^{D-1}+(D-1) z^{D-2} .
$$

The system can be expressed as

$$
\left[\begin{array}{cc}
z_{0}-1 & 1 \\
1 & 0
\end{array}\right]\left[\begin{array}{c}
K_{p} \\
K_{i}
\end{array}\right]=\left[\begin{array}{c}
-C\left(z_{0}\right) \\
-C^{\prime}\left(z_{0}\right)
\end{array}\right] .
$$

Solving for $K_{p}$ and $K_{i}$ yields

$$
\left[\begin{array}{c}
K_{p} \\
K_{i}
\end{array}\right]=\left[\begin{array}{cc}
0 & 1 \\
1 & 1-z_{0}
\end{array}\right]\left[\begin{array}{c}
-C\left(z_{0}\right) \\
-C^{\prime}\left(z_{0}\right)
\end{array}\right] .
$$

\section{Testing For Dominance and Stability}

Out of a total $D+1$ poles, $z_{0}$ and $z_{1}$ are specified (as shown in Section IV) through the choice of $\zeta$ and $\omega_{n}$. For dominant-pole approximations to be accurate, the remaining $D-1$ poles must exist within a radius of $r_{0}=\min \left\{\left|z_{0}\right|,\left|z_{1}\right|\right\}^{A}$ from the origin, where $A$ is the dominance criteria as explained in Section III. This section derives a test that can be used to ensure this accuracy.

Jensen's formula [11] relates an integral of a function $f(z)$ to its $N$ roots $\left\{\alpha_{n}\right\}$ located inside a disk of radius $r$ :

$$
|f(0)| \prod_{n=0}^{N-1} \frac{r}{\left|\alpha_{n}\right|}=e^{\int_{0}^{1} \ln \left|f\left(r e^{j 2 \pi \theta}\right)\right| d \theta} .
$$

For DT-PLLs with delays, Jensen's formula can be applied using the characteristic polynomial $P(z)$ as defined in (5) with roots $\left\{z_{n}\right\}$ to determine if all $D-1$ remaining poles are within the disk of radius $r=r_{0}$. Noting that $z_{0}$ and $z_{1}$ are necessarily outside the disk, and $|P(0)|=\prod_{n=0}^{D}\left|z_{n}\right|=\left|K_{i}-K_{p}\right|$, the left-hand side of (28) simplifies to

$$
\begin{aligned}
|P(0)| \prod_{n=2}^{D} \frac{r_{0}}{\left|z_{n}\right|} & =\frac{\left|K_{i}-K_{p}\right| r_{0}^{D-1}}{\prod_{n=2}^{D}\left|z_{n}\right|} \\
& =\frac{\left|K_{i}-K_{p}\right| r_{0}^{D-1}}{\prod_{n=0}^{D}\left|z_{n}\right| /\left|z_{0} z_{1}\right|} \\
& =\left|z_{0} z_{1}\right| r_{0}^{D-1} .
\end{aligned}
$$

Incorporating this result with the right-hand side of Jensen's inequality results in

$$
\ln \left(\left|z_{0} z_{1}\right| r_{0}^{D-1}\right)=\int_{0}^{1} \ln \left|P\left(r_{0} e^{j 2 \pi \theta}\right)\right| d \theta .
$$

For a choice of $z_{0}$ and $z_{1}$, or, equivalently, $\zeta$ and $\omega_{n} T$, (32) holds when all $D-1$ non-dominant poles are contained within 


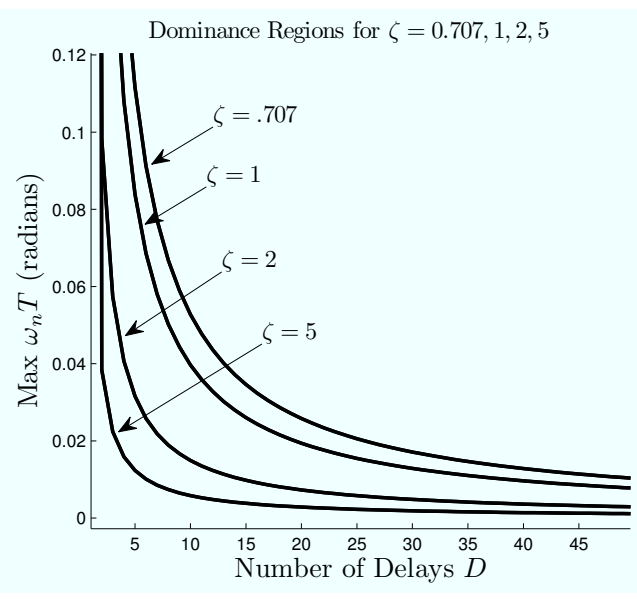

(a)

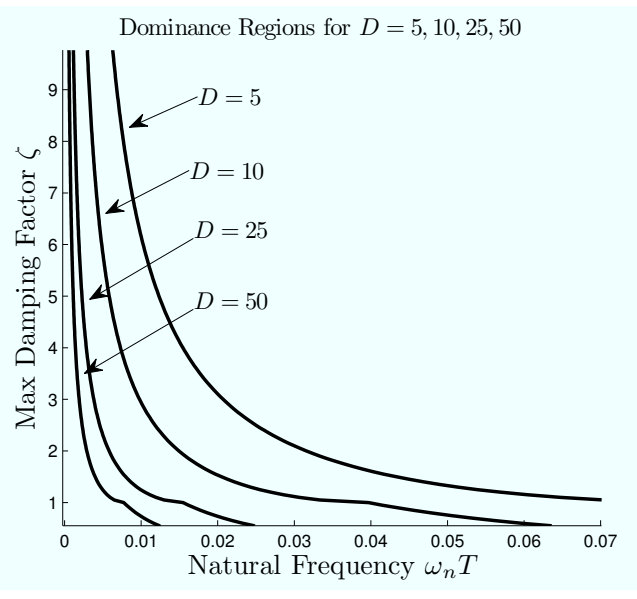

(b)

Fig. 4. Dominant regions computed using (32) for varying $D, \omega_{n} T$, and $\zeta$ with a dominance criteria value of 3: $r_{0}=\min \left\{\left|z_{0}\right|,\left|z_{1}\right|\right\}^{3}$. The dominant regions are the areas below each curve.

a disk of radius $r_{0}=\min \left\{\left|z_{0}\right|,\left|z_{1}\right|\right\}^{A}$. If this is the case, the system is well-approximated and stable. If one or more of the non-dominant poles is located outside the disk, the righthand side of (32) is changed, and the equality is violated. The dominant-pole approximation is considered to be inaccurate, and no guarantee on stability or transient behavior is made.

Note that the dominance test (32) is only valid for cases when $D>1$. When $D=1$, the system is always considered to be dominant since only two poles exist.

Figure 4 shows the regions from which $\zeta$ and $\omega_{n} T$ can be selected for a specified level of dominance. The curves show the points at which (32) is at the verge of non-dominance. Any point below the curve satisfies (32) and thus is well approximated using dominant poles.

The effect of the delays is illustrated in Fig. 4. When the system is underdamped, a higher natural frequency is possible. When the system is overdamped, natural frequency must be low for the system to remain dominant. In other words, maintaining a dominant system is related to the product of natural frequency and damping. As delays increase, the acceptable region is made smaller, and either natural frequency or damping must be reduced to maintain dominance and stability.

Two details of Fig. 4 should be explained. First, discontinuities can be seen in Fig. 4(b) as the damping factor approaches one. This is due to the locus of the two dominant poles as they join on the real axis in transit from the underdamped case to the overdamped case. Secondly, Fig. 4(a) shows that the system is always dominant when $D=1$ due to the reasons explained in Section V.

\section{RESUlTS}

This section illustrates the accuracy of the dominant-pole method in obtaining transient properties closer to those of second-order DT-PLLs. Figure 5 shows an example step response for a true second-order DT-PLL with $\omega_{n} T=.05$, and $\zeta=.707$. The other two responses are for loops with $D=10$ delays, one with parameters derived using the dominant-pole

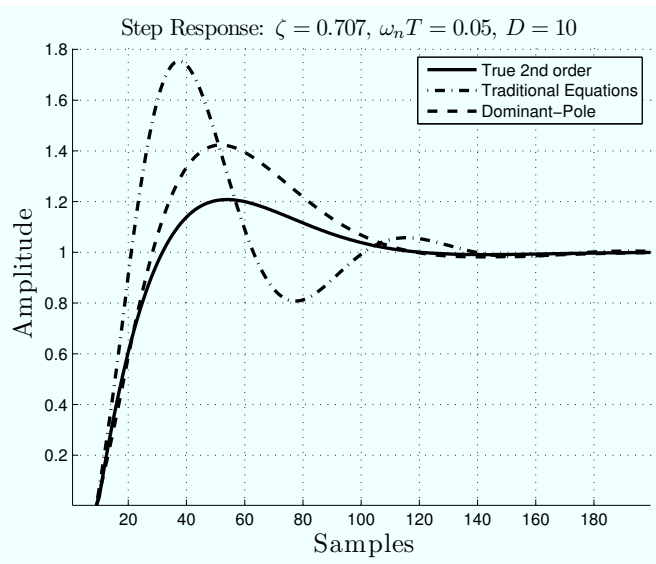

Fig. 5. Step response with parameters derived from traditional (ignoring delays), dominant-pole definitions, and true second-order (without delays) methods. $D=10, \omega_{n} T=.05$, and $\zeta=.707$. In this case, the dominantpole approximation is much more accurate than the traditional equations. Note that the true second-order step response has been initially shifted by $D=10$ samples for easier comparison of transient characteristics.

approximation, and the other from the traditional equations in (2). This example shows that the dominant-pole approximation provides a step response that better matches a second-order response, as the amount of oscillation and settling time match the desired curve.

It is possible for a DT-PLL to be stable, but not wellapproximated using the dominant-pole method. However, the phase margin for these cases is usually low, and should be avoided if possible. An example of this is shown in Fig. 6(b). In these cases, the natural frequency can be lowered until all non-dominant poles are located in the appropriate region.

A key penalty for the delayed feedback is increased overshoot. Additional overshoot is caused by a change in the zero of the transfer function (3), as the values of $K_{p}$ and $K_{i}$ are necessarily different for the second-order and delayed cases. The amount of overshoot is dependent on the desired dominant natural frequency, damping factor, and number of delays. Fig. 7 illustrates the percent overshoot vs. natural 


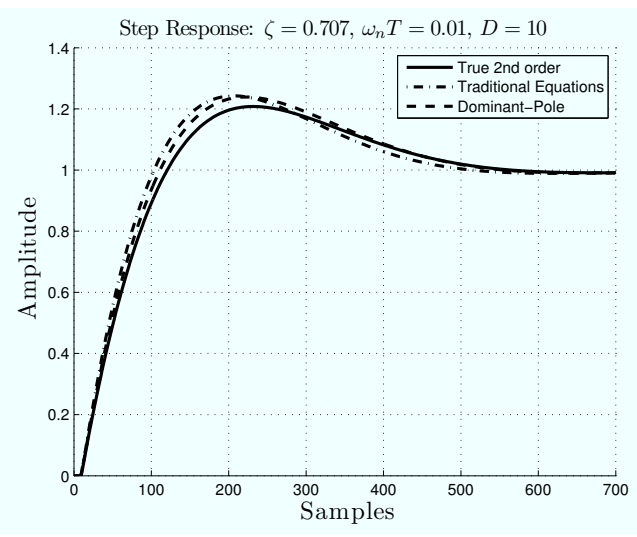

(a)

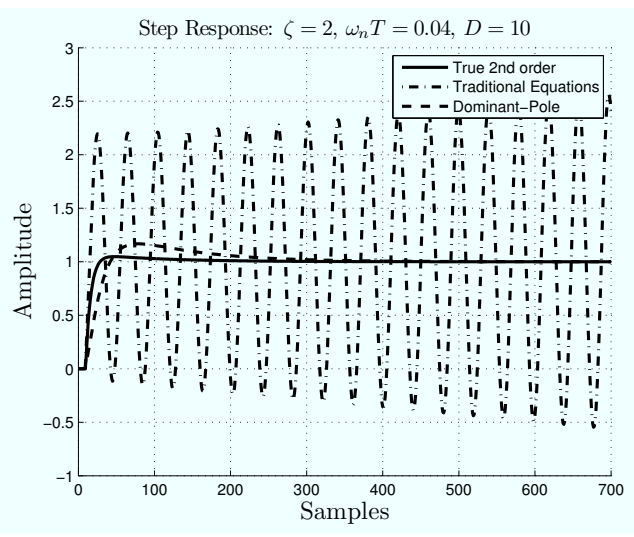

(b)

Fig. 6. Step responses with parameters derived from traditional (ignoring delays), dominant-pole, and true second-order (without delays) methods. Note that the true second-order step response has been initially delayed by $D=10$ samples for easier comparison of transient characteristics. (a) In this case, both the traditional and dominant-pole approximations are very close to the true second-order system. (b) In this case, the traditional equations produce unstable results, and the system is not well-approximated by the dominant-pole approach. This is evident from the noticeable difference in settling time and transient shape.

frequency for $D=10$ and $\zeta=.707$, and the results are similar for other cases of damping and delays. As the natural frequency is reduced, the percent overshoot is also reduced, and the dominant-pole and traditional methods approach the transient characteristics of a true second-order DT-PLL. In other words, if the natural frequency of the loop is sufficiently low, the effect of the delays is negligible and the traditional equations can provide a simpler solution (see Fig. 6(a)).

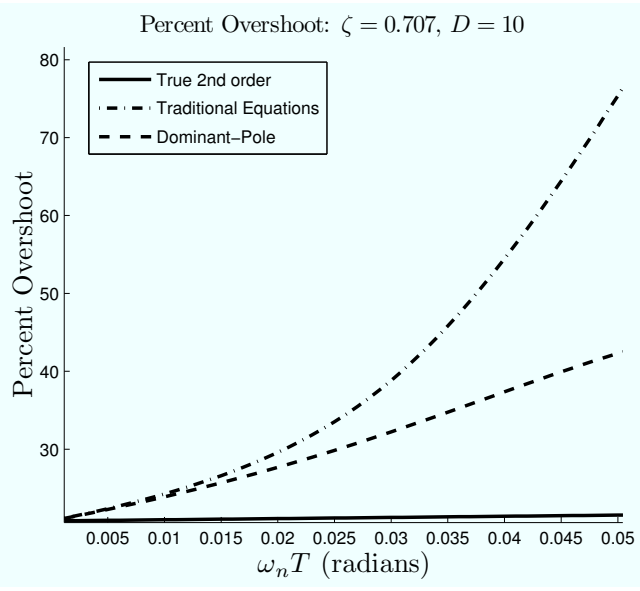

Fig. 7. Percent overshoot vs. $\omega_{n} T$ with parameters derived from traditional (ignoring delays), dominant-pole, and true second-order (without delays) methods. $D=10$ and $\zeta=.707$.

\section{CONCLUSION}

Dominant poles can be used to reduce high-order discretetime phase-locked loops with delays to second-order approximations. It has been shown that loop gain parameters for a type-2 DT-PLL with delays can be directly calculated to provide a particular dominant damping ratio and dominant natural frequency. These values are useful in setting a desired transient behavior of a loop. Furthermore, a numerical integration test has been derived that can be used to ensure stability and accuracy of the dominant-pole approximations.
The novelty of the work presented here is that loop parameters (in the presence of delays) can be calculated without the need for human interaction, simulations, root-finding algorithms, or guess-and-check methods. The simplicity of the solution saves human design time, and allows loop parameters to be automatically calculated under circumstances that were previously impractical. For example, a DT-PLL with any number of delays could automatically change its bandwidth or damping based on real-time measurements, without the risk of instability. A loop's bandwidth could be decreased to clean up a signal in high-noise situations, or increased for faster tracking in mobile scenarios.

\section{REFERENCES}

[1] J. Bergmans, "Effect of loop delay on phase margin of first-order and second-order control loops," IEEE Transactions on Circuits and Systems - II, vol. 52, pp. 621-625, October 2005.

[2] J. Bergmans, "Effect of loop delay on stability of discrete-time PLL," IEEE Transactions on Circuits and Systems - I, vol. 42, pp. 229-231, April 1995.

[3] A. De Gloria, D. Grosso, M. Olivieri, and G. Restani, "A novel stability analysis of a PLL for timing recovery in hard disk drives," IEEE Transactions on Circuits and Systems - I, vol. 48, pp. 1026-1031, August 1999.

[4] F. M. Gardner, Phase-lock Techniques. Hoboken, NJ, USA: John Wiley and Sons, 2005.

[5] A. Spalvieri and M. Magarini, "Wieners analysis of the discrete-time phase-locked loop with loop delay," IEEE Transactions on Circuits and Systems - II, vol. 55, pp. 596-600, June 2008.

[6] A. Patapoutian, "Application of Kalman filters with a loop delay in synchronization," IEEE Transactions on Communications, vol. 50, no. 5, pp. 703-706, 2002.

[7] P. Hanumolu, M. Brownlee, and K. Mayaram, "Analysis of chargepump phase-locked loops," IEEE Transactions on Circuits and Systems I: Regular Papers, vol. 51, no. 9, pp. 1665-1674, 2004.

[8] N. Martins, L. Lima, and H. Pinto, "Computing dominant poles of power system transfer functions," IEEE Transactions on Power Systems, vol. 11, no. 1, pp. 162-170, 1996.

[9] B. Lathi, Linear systems and signals. Oxford University Press, USA, 2002.

[10] R. C. Dorf and R. H. Bishop, Modern Control Systems. Upper Saddle River, NJ, USA: Pearson Prentice Hall, 2005.

[11] W. Rudin, Real and Complex Analysis. New York, New York, USA: McGraw Publishing Company, 1987. 